\title{
EMPREGANDO SITUAÇÕES-PROBLEMA COMO PARTE DOS PROCESSOS DE ENSINO-APRENDIZAGEM DE UMA DISCIPLINA DA ÁREA DE MANUFATURA: RESULTADOS DE AVALIAÇÃO
}

\author{
Armando Í. S. Antonialli - antonialli@ufscar.br \\ Flávio Y. Watanabe - fywatanabe@ufscar.br \\ Carlos E. H. Ventura - ventura@ufscar.br \\ Universidade Federal de São Carlos, Departamento de Engenharia Mecânica \\ Rodovia Washington Luís, km 235 \\ 13.565-905 - São Carlos - SP
}

\begin{abstract}
Resumo: A aprendizagem baseada em problemas refere-se a uma metodologia ativa caracterizada pelo emprego de situações-problema como disparadores à apropriação de conhecimento e desenvolvimento de habilidades desejáveis à formação dos educandos. As situações consistem em narrativas, contextualizadas à futura prática profissional dos estudantes, que instigam a sua curiosidade e criatividade. Na esfera das metodologias ativas, este trabalho encerra uma pesquisa quantitativa sobre os resultados de diferentes instrumentos de avaliação empregados em uma disciplina, cujo escopo envolve processos de fabricação por usinagem, na área de manufatura de um curso de engenharia mecânica. Os resultados mostram que, em se tratando de uma avaliação individual dissertativa, a turma em que foram empregadas situações-problema como parte dos processos de ensinoaprendizagem apresentou desempenho estatisticamente melhor do que a turma em que essa ferramenta não foi utilizada, embora o mesmo não se verifique para os relatórios configurados como instrumento de avaliação por equipe.
\end{abstract}

Palavras-chave: Engenharia mecânica. Processos de fabricação. Usinagem. Metodologias ativas.

\section{INTRODUÇÃO}

Tanto o educando quanto o educador da área de engenharia devem ser capazes de lidar com demandas relacionadas a manufatura e ciclo de vida de produtos, bem como manutenção, mas também com questões globais, jurídicas, de propriedade intelectual, de marketing e relacionadas ao meio-ambiente. Assim sendo, é necessário ao profissional muito mais do que arcabouço técnico, mas também capacidade de interagir com pessoas dos mais diferentes perfis, trabalhar em equipe, liderar projetos, resolver problemas com criatividade, originalidade, ética e comunicar-se bem. El-Madany e Al-Bahkali (2009) apresentam um método interessante de avaliação da percepção dos resultados de aprendizagem por meio de um questionário aplicado aos estudantes de engenharia mecânica ao fim do semestre, por meio do qual foi possível traçar um panorama relacionado à sua formação. A principal conclusão do seu trabalho é que, apesar do extensivo ganho de conhecimentos e habilidades relacionados à identificação, formulação e resolução de problemas, os estudantes apresentam dificuldades em aplicar conhecimentos básicos e também de trabalhar em equipe. 
No ambiente fabril, o desenvolvimento e a implementação de sistemas produtivos flexíveis são fundamentais para atender às flutuações das demandas de mercado, especialmente em relação ao crescente interesse pela customização dos bens de consumo. Nesse contexto, as abordagens inovadoras de ensino-aprendizagem podem auxiliar os futuros engenheiros no que tange à aquisição de diferentes habilidades e competências. Por essa razão, Anderson, Brunoe e Nielsen (2019) propõem que o projeto pedagógico de um curso de graduação em engenharia contenha estratégias como aprendizagem baseada em problemas (ABP ou PBL, do inglês problem-based learning) e fábricas de aprendizagem (Learning factories), além do uso massivo de ferramentas digitais e ensino híbrido (Blended learning). Dessa forma, segundo os autores, é possível que haja a interação entre os conhecimentos tecnológico, pedagógico e de conteúdo, conforme apresentado na Figura 1.

Figura 1 - A integração entre os conhecimentos tecnológico (T), pedagógico (P) e de conteúdo (C).

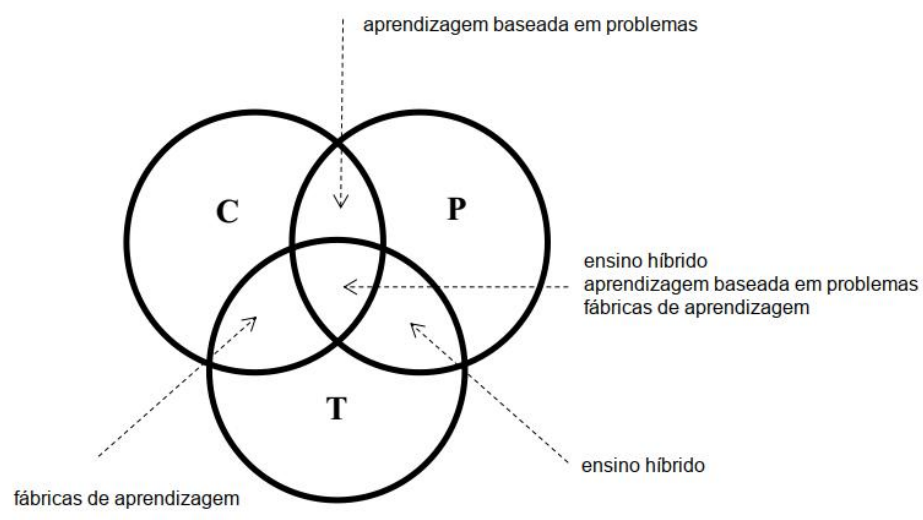

Fonte: Anderson, Brunoe e Nielsen (2019).

Uma característica importante da $\mathrm{ABP}$ é o fato de uma situação-problema sempre preceder a apresentação dos conceitos necessários para a sua resolução, de maneira que ela sirva de disparadora e motivadora para a aprendizagem de conteúdos específicos, bem como promotora do desenvolvimento de habilidades e atitudes profissional e socialmente desejáveis. Adicionalmente, a ABP é caracterizada pelo trabalho dos estudantes em pequenos grupos facilitados por tutores (ESCRIVÃO FILHO; RIBEIRO, 2008).

Hassan et al. (2012) indicam a aprendizagem cooperativa baseada em problemas como estratégia para contribuir com o ganho de maturidade dos estudantes de engenharia química, visto que a solução de problemas em si corresponde a um processo cognitivo de alta ordem que acessa as mais complexas funções intelectuais. Cascini et al. (2015), por sua vez, sugerem que a estratégia de gamificação baseada na Teoria da Solução Inventiva de Problemas (OSTM-TRIZ) pode ser usada de forma eficiente no estímulo à criatividade dos estudantes de engenharia, proporcionando reflexões interessantes durante a resolução das situações e tarefas absolutamente novas. Os índices de criatividade demonstram melhoria significativa, assim como os indicadores relacionados a habilidades mentais. De qualquer forma, a generalização dessa conclusão deve ser cuidadosa, dada a limitação do estudo. Joshi, Desai e Tewari (2020) combinaram a aprendizagem baseada em problemas com a análise da aprendizagem por meio da tecnologia (Learning analytics), de maneira a levantar e avaliar dados para subsidiar a tomada de decisões, apresentando benefícios como a possibilidade de ofertar atividades curriculares mais direcionadas, o retorno rápido aos estudantes, o seu desenvolvimento em termos de comportamento e desempenho, bem como o aprofundamento da aprendizagem customizada e a possibilidade de aprimoramento do currículo. Adicionalmente, destacam-se o 
aumento da autonomia do estudante e da sua conscientização acerca da necessidade da aprendizagem continuada.

Embora a efetividade da ABP esteja bem sustentada quanto ao desenvolvimento do pensamento crítico e da capacidade dos estudantes em solucionar conflitos, ainda há dúvidas em relação ao seu impacto sobre a fixação de conteúdo. Por essa razão, Carriger (2015) propõe uma abordagem montessoriana da aprendizagem baseada em problemas, de maneira que os objetivos de aprendizagem pudessem ser focados não no domínio de conteúdo, mas na capacidade de aplicá-lo. Essa nova perspectiva demandaria o desenvolvimento adicional de habilidades metacognitivas e, consequentemente, alterações nos processos de avaliação e na estrutura do currículo do curso. De qualquer forma, seria um modelo aplicável a atividades curriculares em estágios mais avançados de um curso de graduação, ao passo que as aulas expositivas tradicionais poderiam ser eficientemente aplicadas às disciplinas introdutórias, com as ciências básicas.

Considerando, em particular, o interesse pelo efeito das características dos "Millennials" (ou da Geração Z) sobre os processos de ensino-aprendizagem, Hsieh e Knight (2008) descrevem dois estudos, um piloto e outro mais aprofundado, que comparam a ABP com o ensino tradicional expositivo empregado com calouros de engenharia a partir de instrumentos de avaliação que consideravam os diferentes perfis de aprendizagem. A despeito das limitações desse trabalho, as autoras concluem que a aprendizagem baseada em problemas pode ser uma abordagem pedagógica efetiva para superar a dificuldade de transposição entre teoria e prática na formação dos engenheiros, além de contribuir como um fator motivacional no início do curso.

Ainda que a aprendizagem baseada em problemas se relacione, tipicamente, com currículos não-disciplinares e com trabalho em pequenos grupos, Pastirik (2006) destaca uma experiência de aplicação dessa estratégia também em grandes grupos, em que se alternavam momentos de orientação particularizada para pequenas subdivisões do mesmo, empregando ainda tecnologias digitais de informação e comunicação. Ainda que seja relatada a dificuldade de não se poder contar com auxiliares para a mediação e avaliação dos grupos menores, a autora destaca que mudanças na estrutura mais canônica da ABP podem ser viáveis e efetivas nos processos de ensino-aprendizagem, em consonância com ferramentas digitais, por exemplo.

Tendo em vista incertezas e possíveis benefícios da aplicação da ABP, o presente trabalho se refere a uma experiência de utilização de situações-problema como parte dos processos de ensino-aprendizagem de uma disciplina do curso de graduação em engenharia mecânica da Universidade Federal de São Carlos (UFSCar) e traz resultados quantitativos de desempenho dos alunos submetidos a este método em comparação a um grupo controle.

\section{METODOLOGIA}

O curso de graduação em engenharia mecânica da UFSCar foi criado em 2008 no âmbito do Programa de Apoio a Planos de Reestruturação e Expansão das Universidades Federais (REUNI) para receber seus primeiros 45 ingressantes em 2009, ampliando a oferta de vagas anuais para 60 estudantes, através do Sistema de Seleção Unificada (SiSU), a partir de 2015; adicionam-se, ainda, duas vagas anuais pelo Vestibular Indígena e pelo Ingresso para Refugiados, sem contar os Processos de Transferência Interna e Externa. Seu projeto pedagógico (UNIVERSIDADE FEDERAL DE SÃO CARLOS, 2019) destaca, dentre outras especificidades, as competências, habilidades e atitudes, além dos valores fundamentais a serem adquiridos durante o curso. Com relação às competências, em especial, existe uma 
aderência evidente com os tipos de problemas a serem resolvidos pelos engenheiros, bem como seus contextos de atuação.

A disciplina "Princípios de Usinagem" é uma atividade curricular obrigatória, oferecida aos estudantes do sexto período, dentro do Núcleo de Formação Específica no módulo de Processos de Fabricação Mecânica, usualmente denominado como área de Manufatura. Sua ementa envolve conceitos básicos, como: geometria da cunha de corte e mecanismo da formação do cavaco; cálculo de forças e potências; materiais para ferramentas; avarias, desgastes e vida das ferramentas; noções sobre lubrificação e refrigeração; condições de economia e de máxima produção; integridade superficial e usinabilidade dos metais.

Durante o segundo semestre de 2017, o Departamento de Engenharia Mecânica (DEMec/UFSCar) ofertou duas turmas da referida disciplina em horários distintos. Em virtude disso, houve 31 estudantes deferidos em uma turma (denominada Turma A), e apenas 10 na outra (denominada Turma B). A existência de uma turma atipicamente reduzida instigou o docente responsável a considerar a adoção de metodologias ativas. Nesse sentido, foram elaboradas duas situações-problema para que fossem empregadas como parte do processo de ensino-aprendizagem da Turma B nos tópicos "mecanismo da formação do cavaco" e "cálculo de forças e potências", enquanto os mesmos foram apresentados à Turma A na forma tradicional de aula expositiva dialogada.

As situações-problema em questão consistiram em breves narrativas fictícias e originais envolvendo diálogos que remetiam a um ambiente da indústria metalmecânica, nos quais o personagem estagiário era chamado a resolver um problema apontado pelos seus superiores, sendo cada problema relacionado a um dos tópicos supramencionados. Após a leitura teatralizada da situação-problema, os estudantes foram instigados a esclarecer termos eventualmente desconhecidos. Na sequência, deveriam formular hipóteses por meio de brainstorming e construir questões de aprendizagem que pudessem sustentá-las por meio de uma síntese provisória. Borges et al. (2014) descrevem essa metodologia passo a passo. Finalizando a primeira aula, os estudantes eram orientados a responder àquelas questões durante um momento de estudo individual, fora do horário da disciplina. Na aula seguinte, os estudantes deveriam rediscutir o problema em face aos novos conhecimentos adquiridos e construir uma síntese definitiva.

A avaliação somativa da disciplina para ambas as turmas era constituída de um miniprojeto e relatórios de aulas práticas como instrumentos por equipe; e avaliações dissertativas (com consulta) como instrumentos individuais. Os dois tópicos mencionados foram contemplados em dois relatórios e uma avaliação dissertativa. A todos eles eram atribuídas notas individuais de zero a dez. O desempenho das duas turmas em termos desses três instrumentos de avaliação foi comparado através do teste t de Student, calculado por meio da Equação 1, em que $\bar{y}_{1 j}$ e $\bar{y}_{2 j}$ são as médias das notas do instrumento $j$ para cada uma das duas turmas, considerando $S_{p j}^{2}$ a estimativa da variância das mesmas e $n_{1}$ e $n_{2}$ o tamanho das turmas (MONTGOMERY, 2017).

$$
\mathrm{t}_{\mathrm{j}}=\frac{\overline{\mathrm{y}}_{1 \mathrm{j}}-\overline{\mathrm{y}}_{2 \mathrm{j}}}{\mathrm{S}_{\mathrm{pj}} \sqrt{1 / \mathrm{n}_{1}+1 / \mathrm{n}_{2}}}
$$

A estimativa da variância das notas para cada instrumento $j\left(S_{p j}^{2}\right)$ deve ser calculada pela Equação 2, em que $S_{1 j}^{2}$ e $S_{2 j}^{2}$ são as variâncias das notas em cada uma das turmas.

$$
S_{p j}^{2}=\frac{\left(\mathrm{n}_{1}-1\right) \mathrm{S}_{1 \mathrm{j}}^{2}+\left(\mathrm{n}_{2}-1\right) \mathrm{S}_{2 \mathrm{j}}^{2}}{\mathrm{n}_{1}+\mathrm{n}_{2}-2}
$$


Pode-se dizer que as médias das turmas para certo instrumento $j$ são diferentes se o valor absoluto do teste $t j$ for maior que o valor da distribuição $\mathrm{t}$ com $n_{1}+n_{2}-2$ graus de liberdade para um dado nível de significância $\alpha$, que, neste caso, corresponde a 2,023 em se tratando de um intervalo de confiança de $95 \%$. O mesmo raciocínio é válido para comparar o desempenho acadêmico geral das duas turmas considerando a média ponderada de cada um dos estudantes em todas as atividades curriculares acadêmicas.

\section{RESULTADOS E DISCUSSÃO}

A Figura 2 ilustra o resultado dos três instrumentos empregados na avaliação somativa da disciplina para os dois tópicos mencionados anteriormente, a saber: os Relatórios 1 e 2 e uma Avaliação dissertativa. A todos eles foram atribuídas notas individuais de zero a dez. As colunas verticais se referem às médias aritméticas calculadas a partir das notas dos estudantes da Turma A, em que os tópicos foram abordados da forma tradicional de aula expositiva dialogada (cinza claro), e da Turma B, em que foram empregadas situações-problema como parte do processo de ensino-aprendizagem (cinza escuro). As barras de erro apresentam os desvios padrão em torno das referidas médias.

Figura 2 - Resultados dos diferentes instrumentos de avaliação empregados.

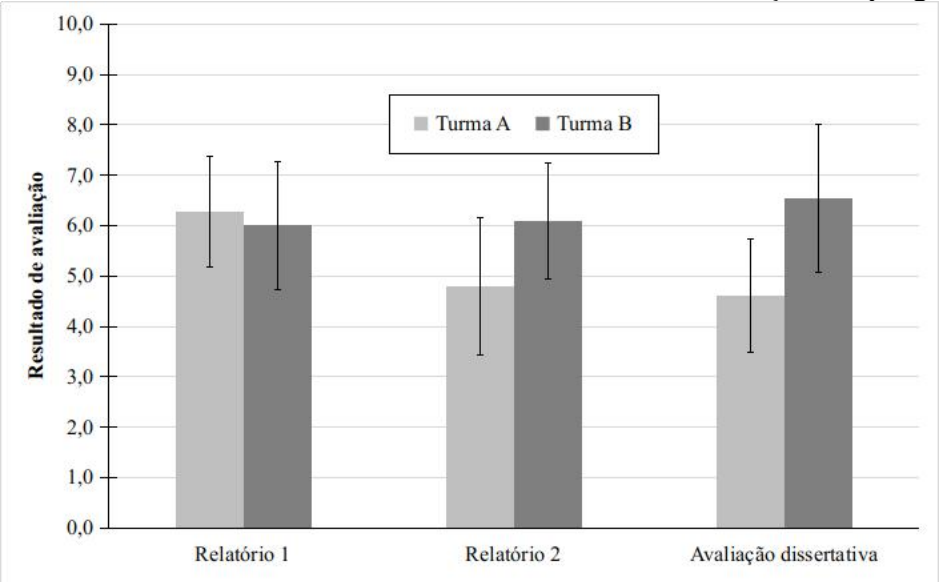

Fonte: Elaborada pelos autores.

É possível perceber que as médias são muito próximas no caso do Relatório 1: 6,3 para a Turma A e 6,0 para a Turma B. A diferença é um pouco maior, mas para o outro lado, no caso do Relatório 2: 4,8 para a Turma A e 6,1 para a Turma B. Para a Avaliação dissertativa, finalmente, as médias se mostram mais distantes: 5,0 para a Turma A e 8,5 para a Turma B. O desvio padrão se mantém elevado (entre 2,2 e 3,0), de uma maneira geral, para ambas as turmas nos três instrumentos de avaliação. Era importante identificar se a diferença entre essas médias seria estatisticamente significante ou não. Assim sendo, o desempenho das Turmas A e B, em termos dos instrumentos "Relatório 1", "Relatório 2" e "Avaliação dissertativa" foi comparado por meio do teste $t$ de Student, conforme apresentado na Figura 3. 
Figura 3 - Teste t de Student (valor absoluto) para as turmas em relação aos diferentes instrumentos de avaliação.

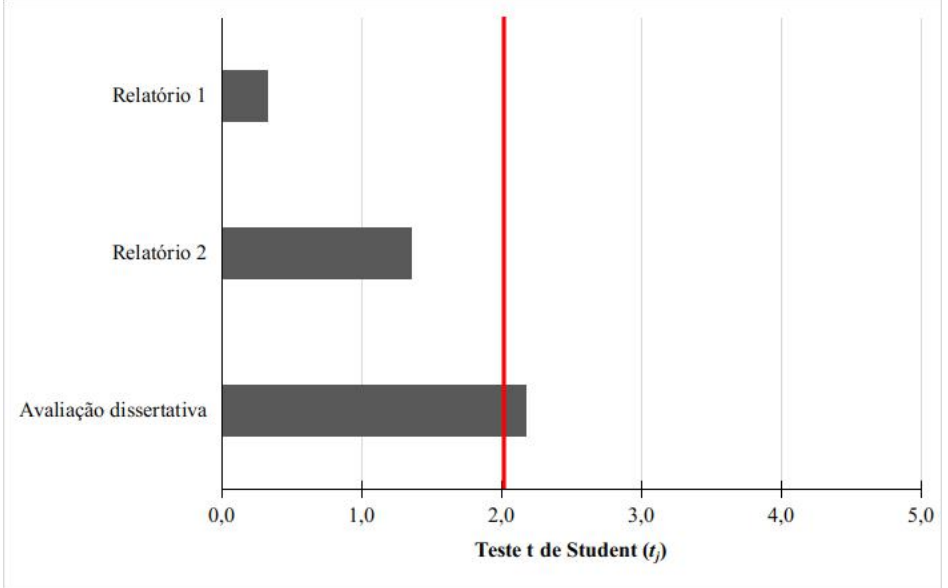

Fonte: Elaborada pelos autores.

Como pode ser visto, no caso dos dois relatórios, o valor absoluto do teste, apresentado como barras horizontais (0,329 para o Relatório 1 e 1,364 para o Relatório 2), se mostra inferior ao valor da distribuição t com 39 graus de liberdade e significância $5 \%(2,023)$, ilustrado pela linha vermelha. No entanto, o valor absoluto do teste $(2,180)$ é maior que o referido valor da distribuição no caso da avaliação dissertativa.

Em termos quantitativos, é correto dizer que não se pode rejeitar a hipótese nula, de que as médias das turmas seriam estatisticamente iguais, no caso dos instrumentos de avaliação "Relatório 1" e "Relatório 2". Por outro lado, deve-se rejeitar a hipótese nula para a "Avaliação dissertativa" e, portanto, é possível dizer que a média da Turma B é estatisticamente superior à média da Turma A considerando um intervalo de confiança de $95 \%$.

Embora o desvio padrão em torno das médias das notas das duas turmas, nos três instrumentos, seja consideravelmente elevado, a abordagem estatística é clara e inequívoca: o desempenho da Turma B é superior ao desempenho da Turma A na avaliação dissertativa. É claro que se poderia associar essa diferença à inegável vantagem de ter sido possível trabalhar com um grupo reduzido de estudantes naquela turma e, assim, promover relações de ensinoaprendizagem mais estreitas. Contudo, é razoável também inferir que o emprego de situaçõesproblema seja uma importante razão pela qual a média da referida turma foi superior à média da outra, embora essa diferença não se tenha manifestado para os outros instrumentos de avaliação.

Levando-se em consideração os apontamentos de Hassan et al. (2012), sobre o aumento de maturidade, e de Joshi, Desai e Tewari (2020), sobre o aumento de autonomia dos estudantes que foram expostos à aprendizagem baseada em problemas, é possível supor que características como essas tenham sido talvez mais determinantes para um bom desempenho em uma avaliação dissertativa individual do que em relatórios de atividades práticas realizados em equipe. Vale destacar que a avaliação dissertativa foi realizada com consulta e, portanto, estaria direcionada a um processo cognitivo de mais elevada ordem.

Uma observação adicional, importante para concluir a discussão, refere-se ao desempenho acadêmico geral das duas turmas considerando a média ponderada de cada um dos estudantes em todas as atividades curriculares acadêmicas realizadas até aquele momento. Afinal de contas, se as turmas não fossem minimamente similares nesse sentido, qualquer discussão em torno da efetividade das situações-problema enquanto estratégia de ensinoaprendizagem estaria comprometida. A média ponderada acadêmica geral e o desvio padrão associado foram calculados para as duas turmas e estão apresentados na Tabela 1, bem como 
o valor absoluto do teste t nesse caso: 0,265 e, portanto, inferior ao valor da distribuição t com 39 graus de liberdade e significância $5 \%(2,023)$. Dessa forma, não se pode refutar a hipótese de que o desempenho geral das turmas seja estatisticamente igual e, assim, a proposição de que o emprego de situações-problema possa vir a proporcionar resultados de avaliação mais interessantes do que uma aula tradicional expositiva permanece válida.

Tabela 1 - Desempenho acadêmico geral das duas turmas (considerando todas as atividades curriculares acadêmicas realizadas pelos estudantes).

\begin{tabular}{ccccc}
\hline & Média & Desvio padrão & Teste $\mathrm{t}$ & Conclusão \\
\hline Turma A & 6,6 & 1,0 & $0,265(<2,023)$ & O mesmo desempenho acadêmico geral \\
Turma B & 6,5 & 1,4 & & \\
\hline
\end{tabular}

Fonte: Elaborada pelos autores.

\section{CONSIDERAÇÕES FINAIS}

Antes de mais nada, é fundamental apontar que a metodologia de aprendizagem baseada em problemas (ABP) envolve uma arquitetura curricular muito mais abrangente do que a simples adoção de uma dada ferramenta dentro de uma certa disciplina. Por essa razão, os autores tiveram o cuidado de não associar este trabalho a uma experiência de ABP, embora, por razões óbvias, a referida estratégia apareça com destaque na revisão da literatura.

A oportunidade de empregar situações-problema em um curso disciplinar tradicional foi encorajada pela sorte de contar com uma turma com número atipicamente reduzido de estudantes no período ao qual se refere este trabalho. Considerando os bons resultados apresentados por essa iniciativa, entendida como altamente promissora, a estratégia foi repetida, nos semestres seguintes, mesmo em turmas maiores (com mais de 50 estudantes), de forma similar àquela apresentada por Pastirik (2006).

Nessas ocasiões, após a leitura da narrativa, as turmas eram divididas dentro do espaço da sala de aula em pequenos grupos (com dez estudantes, em média), os quais eram instrumentalizados com uma sequência de instruções para que pudessem se auto-organizar sem a necessidade de facilitação permanente do docente. Dentre elas, a necessidade de eleger um coordenador, que seria responsável pela gestão do tempo para a execução das atividades da agenda, e um relator, para registrar os principais pontos da discussão, bem como as hipóteses e questões de aprendizagem levantadas. $\mathrm{Na}$ aula seguinte, com a sala na disposição mais convencional, o docente utilizava a síntese provisória de todos os pequenos grupos para conduzir a exposição dialogada, procurando, em conjunto, confirmar ou refutar as hipóteses enumeradas por eles. A avaliação do desempenho das turmas nessa nova modalidade de aplicação de situações-problema será objeto de trabalhos futuros.

\section{Agradecimentos}

Os autores gostariam de agradecer ao Grupo de Trabalho em Metodologias Ativas e Avaliação (MetAA/UFSCar) pela oferta de oficinas de formação docente como resposta às demandas de apropriação de novas práticas pedagógicas, bem como às necessidades e características próprias das novas gerações de estudantes. 


\section{REFERÊNCIAS}

ANDERSEN, A.-L.; BRUNOE, T. D.; NIELSEN, K. Engineering Education in Changeable and Reconfigurable Manufacturing: Using Problem-Based Learning in a Learning Factory Environment. Procedia CIRP, v. 81, p. 7-12, 2019.

BORGES, M. C. et al. Aprendizado baseado em problemas. Medicina (Ribeirão Preto), v. 47, n. 3, p. 301-317, 2014.

CARRIGER, M. S. Problem-based learning and management development - Empirical and theoretical considerations. The International Journal of Management Education, v. 13, n. 3, p. 249-259, 2015.

CASCINI, G. et al. OTSM-TRIZ Games: Enhancing Creativity of Engineering Students. Procedia Engineering, v. 131, p. 711-720, 2015.

EL-MADANY, M. M.; AL-BAHKALI, E. Mechanical Engineering Design at King Saud University: An Outcome Assessment. Journal of King Saud University - Engineering Sciences, v. 21, n. 1, p. 33-41, 2009.

ESCRIVÃO FILHO, E.; RIBEIRO, L. R. C. Aprendendo com PBL: aprendizagem baseada em problemas: relato de uma experiência em cursos de engenharia da EESC-USP. In: Ciclo de palestras "O ensino no campus da USP - São Carlos: inovações e inovadores", 2008, São Carlos. Anais. São Carlos, 2008.

HASSAN, S. A. H. S. et al. Methods to Study Enhancement of Problem Solving Skills in Engineering Students through Cooperative Problem-Based Learning. Procedia - Social and Behavioral Sciences, v. 56, n. 8, p. 737-746, 2012.

HSIEH, C.; KNIGHT, L. Problem-Based Learning for Engineering Students: An EvidenceBased Comparative Study. The Journal of Academic Librarianship, v. 34, n. 1, p. 25-30, 2008.

JOSHI, A.; DESAI, P.; TEWARI, P. Learning Analytics framework for measuring students' performance and teachers' involvement through problem based learning in engineering education. Procedia Computer Science, v. 172, p. 954-959, 2020.

MONTGOMERY, D. C. Design and Analysis of Experiments. $9^{\text {a }}$ edição, Hoboken: John Wiley \& Sons, 2017.

PASTIRIK, P. Using problem-based learning in a large classroom. Nurse Education in Practice, v. 6, n. 5, p. 261-267, 2006.

UNIVERSIDADE FEDERAL DE SÃO CARLOS. Projeto Pedagógico Curso de Bacharelado em Engenharia Mecânica. Disponível em: http://www.mecanica.ufscar.br/. Acesso em: 30 jun. 2019. 


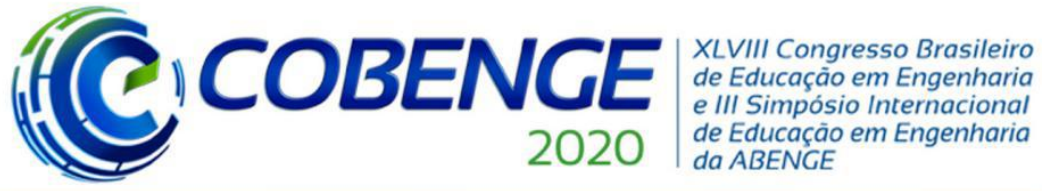

"Os desafios para formar hoje o engenheiro do amanhã"

\title{
EMPLOYING PROBLEM SITUATIONS AS PART OF TEACHING AND LEARNING PROCESS IN A MANUFACTURING COURSE: ASSESSMENT RESULTS
}

\begin{abstract}
Problem-based learning refers to an active learning strategy which employs problem situations as triggers to the access of knowledge and development of well desirable abilities to training and formation. These situations consist in contextualized narratives to the future professional activity of the students which can instigate their curiosity and creativity. On the context of active learning, this work contains a quantitative research about the results of different assessment tools that were applied in a curricular activity whose scope involves machining processes, at the manufacture area of an undergraduate course on mechanical engineering. The results show that, when talking about an individual open-questions evaluation, the class in which problem situations were inserted as part of the teaching and learning process statistically presented a better performance than that in which this strategy was not employed, although it cannot be said the same about the reports that were used as assessment tools for teams evaluating.
\end{abstract}

Keywords: Mechanical engineering. Manufacturing processes. Machining. Active methodologies. 